\title{
三苯胺类自供能电致变色材料合成及器件开发
}

\author{
陈梅 ${ }^{a} \quad$ 杨树威 ${ }^{b}$ 郑建明 $b \quad$ 徐春叶*,a,b \\ ( ${ }^{a}$ 中国科学技术大学 化学与材料科学学院高分子科学与工程系 合肥 230026) \\ $\left({ }^{b}\right.$ 中国科学技术大学 合肥微尺度物质科学国家实验室＼cjkstart合肥 230026)
}

\begin{abstract}
摘要 设计并合成了一种新型三苯胺衍生物：(4-((4-(二甲基氨基)苯基)(苯基)氨基)芐基)膦酸, 并研究了它的光谱电化 学性质和光伏性质. 光谱电化学谱图表明该化合物具有电致变色性能: 通过施加不同电压, 该化合物薄膜可以在透明 态和着色态之间转换, 且在 $629 \mathrm{~nm}$ 处透过率对比度达到最大, 为 $64.2 \%$. 由该材料组装成的自供能电致变色器件具有 光伏性能, 光电转化效率可以达到 $0.32 \%$. 和传统的光电变色器件相比, 该新型器件具有结构简单、制备成本低廉、绿 色环保等特点, 预计在建筑、汽车、显示器等领域将有广泛的应用.
\end{abstract}

关键词 三苯胺衍生物; 光伏; 电致变色; 光电变色器件; 自供能电致变色器件

\section{Synthesis of a Novel Triphenylamine Derivative and Exploration of Self-powered Electrochromic Device

\author{
Chen, $\mathrm{Mei}^{a} \quad$ Yang, Shuwei $^{b} \quad$ Zheng, Jianming $^{b} \quad \mathrm{Xu}$, Chunye* ${ }^{*, a, b}$ \\ $\left({ }^{a}\right.$ Department of Polymer Science and Engineering, School of Chemistry and Materials Science, University of \\ Science and Technology of China, Hefei 230026, China) \\ $\left({ }^{b}\right.$ Hefei National Laboratory for Physical Sciences at Microscale, University of Science and Technology of China, \\ Hefei 230026, China)
}

\begin{abstract}
Self-powered electrochromic device is a kind of instrument that can change transmittance with the electricity converting from solar energy. To realise the dual functions of solar energy harvesting and transmittance changing in one material, a novel derivative of triphenylamine (4-((4-(dimethylamino)phenyl)(phenyl)amino)benzyl)phosphoric acid $\left(\mathrm{Ph}_{2} \mathrm{ABPA}-\mathrm{Me}_{2}\right)$ is designed and synthesized in this paper. ${ }^{1} \mathrm{H}$ NMR, FT-IR and MS were used to characterize the structure of the compound. Working electrode was made by dissolving the compound in chloroform and then immersing titanium dioxide-coated fluorine-doped tin oxide glass in the solution for $24 \mathrm{~h}$. Its spectroelectrochemical properties were investigated with a UV-vis-IR spectrophotometer and a potentiostat, which allowed us to acquire transmittance spectra under potential control in a solution of $0.1 \mathrm{~mol} / \mathrm{L}$ lithium perchlorate in propylene carbonate. The experiment was carried out with three electrodes system in which Ag wire acted as reference electrode and Pt wire as counter electrode. The working electrode shows light yellow with relatively high transmittance at $-1.5 \mathrm{~V}$ and dark green with low transmittance at $+2.5 \mathrm{~V}$. The maximum transmittance contrast between the two states is $64.2 \%$ at $629 \mathrm{~nm}$. Meanwhile, to investigate the photovoltaic properties of $\mathrm{Ph}_{2} \mathrm{ABPA}-\mathrm{Me}_{2}, \mathrm{Ph}_{2} \mathrm{ABPA}-\mathrm{Me}_{2}$-based electrochromic devices were made by sandwiching the working electrode and Pt-based counter electrode together with electrolyte of Iodolyte AN-50. The photovoltaic properties were measured with a solar simulator under 1 sun AM1.5G illumination. Energy conversion efficiency of $0.32 \%$ was obtained, with short-circuit current density of $1.32 \mathrm{~mA} / \mathrm{cm}^{2}$, open-circuit voltage of $0.44 \mathrm{~V}$ and fill factor of 0.54 . All the results mentioned above suggest that the compound has dual functions of electrochromism and photovoltaics. Compared with traditional photoelectrochromic devices with both a photovoltaic electrode and an electrochromic electrode, the dual functional $\mathrm{Ph}_{2} \mathrm{ABPA}-\mathrm{Me}_{2}$-based electrochromic device shows properties of simple construction, cheap to make and friendly to environment, and thus may have potential applications in buildings, vehicles and numerous types of displays.
\end{abstract}

Keywords triphenylamine derivative; photovoltaic; electrochromism; photoelectrochromic devices; self-powered device

\section{1 引言}

随着经济的发展，不可再生能源日益被消耗，新能 源尤其是太阳能的开发和利用迫在眉睫. 太阳能作为地 球上最丰富的绿色能源, 每秒钟照射到地球上的能量就
相当于 500 万吨煤完全燃烧释放的能量，但是利用率低 下阻碍了太阳能的广泛使用，因此提高太阳能的利用率 和节约能源变得尤为重要. 近年来，自供能电致变色窗 越来越受到科学家们的瞩目. 它是一种可以在太阳光的 照射下改变自身颜色, 从而改变透过率的器件. 这类器

*E-mail: chunye@ustc.edu.cn; Tel.: 0551-63603459

Received January 17, 2013; published March 5, 2013.

Project supported by the Hundred Talents Program of Chinese Academy of Sciences, the Thousand Talents Program of Chinese Central Goverment and the National Natural Science Foundation of China (Nos. 21274138, 21074125).

项目受中国科学院百人计划、中组部“千人计划”和国家自然科学基金(Nos. 21274138, 21074125)资助. 
件有如下优点: (1)不需要外加电压就可改变窗户的透过 率, 节约能源; (2)使用清洁的太阳能, 更加环保. 我们 经过不解的探索, 提出两种方案来实现这个目标. 一种 是将染料敏化太阳能电池技术和电致变色技术结合的 光电变色器件 ${ }^{[1]}$, 其器件结构主要包括三个部分, 分别 是具有光伏性能和电致变色性能的两种薄膜组成的两 个电极和电解液, 这部分工作在我们课题组以前的文 章 ${ }^{[2]}$ 里有介绍; 另一种方案是合成一种同时具有电致变 色 ${ }^{[3]}$ 和光伏性能的化合物, 并以此化合物为功能性材料 组装成自供能电致变色器件, 由这种双重功能的材料组 装的器件和上述第一种光电变色器件相比, 结构更加简 单, 光伏性能和电致变色性能由同一种材料实现, 因此 电极的制备过程得到了简化, 由此得到的器件因而具有 结构简单、制备成本低廉、绿色环保等特点.

本研究目的在于开发一种新型的同时具有光伏性 能和电致变色性能的自供能电致变色材料. 通过文献调 研, 我们发现三苯胺是一种非平面的具有螺旋桨式空间 结构的芳胺分子. 其衍生物因良好的光电性能 ${ }^{[4,5]}$ 而被 广泛地用作染料敏化太阳能电池的染料. 2010 年, 王鹏 课题组 ${ }^{[6]}$ 设计合成了以三苯胺作为电子给体的染料C219, 其光电转化效率可以达到 $10.0 \% \sim 10.3 \%$. 2011 年, Lee 等 ${ }^{[7]}$ 报道了一系列新型 $\pi$-共轭有机染料, 其最大 光电转化效率可以达到 $7.30 \%$. 另外, 三苯胺及其衍生 物凭借其良好的热稳定性 ${ }^{[8]}$ 和电化学稳定性 ${ }^{[9]}$ 在电致变 色领域也占有重要的地位. 2011 年, Yen 等 ${ }^{[10]}$ 报道了一 系列三苯胺基芳环聚酰胺. 这些聚合物具有可逆的氧化 还原电势、稳定的电致变色性能, 而且在近红外区和可 见区都有较高的对比度. 但是同时具有光伏性能和电致 变色性能的三苯胺类小分子化合物却少有报道.

本文设计并合成了化合物(4-((4-(二甲基氨基)苯 基)(苯基)氨基)苠基)膦酸 $\left(\mathrm{Ph}_{2} \mathrm{ABPA}-\mathrm{Me}_{2}\right)$. 通过核磁、红 外和质谱确定了其结构. 光谱电化学测试表明该化合物 具有电致变色性能. 为了探究其光伏性能, 我们将其制 作成自供能电致变色器件, 测得其光电转换效率为 $0.32 \%$. 可以预测, 基于 $\mathrm{Ph}_{2} \mathrm{ABPA}-\mathrm{Me}_{2}$ 这种材料的新型 自供能电致变色器件将有望应用于建筑、汽车和各种显 示器领域.

\section{2 结果与讨论}

\section{1 化合物的合成}

\section{1 .1 化合物 $\mathbf{1}$ 的合成}

在惰性气体保护下, 称量 $2.73 \mathrm{~mL}(30 \mathrm{mmol})$ 苯胺、 $4.066 \mathrm{~g}$ (20 mmol) 4-溴- $N, N$-二甲基苯胺、0.12 g (0.13 $\mathrm{mmol}$ )三(二亚苠基丙酥)二钯、 $0.0531 \mathrm{~g}(0.26 \mathrm{mmol})$ 三叔 丁基磷、1.922 g (20 mmol)叔丁醇钠加入甲苯中. $90{ }^{\circ} \mathrm{C}$ 下反应 $24 \mathrm{~h}$. 减压除去甲苯, 用氯仿溶解. 过滤之后, 用饱和食盐水洗涤 3 次. 有机层干燥后过滤, 旋干, 通 过柱层析得到黄色产物(3.162 g, 78\%). ${ }^{1} \mathrm{H}$ NMR (400<smiles>CN(C)c1ccc(Nc2ccc(Br)cc2)cc1</smiles><smiles>CN(C)CC(C)(C)C</smiles><smiles>CN(C)c1ccc(N(c2ccccc2)c2ccc(CO)cc2)cc1</smiles>

(d)
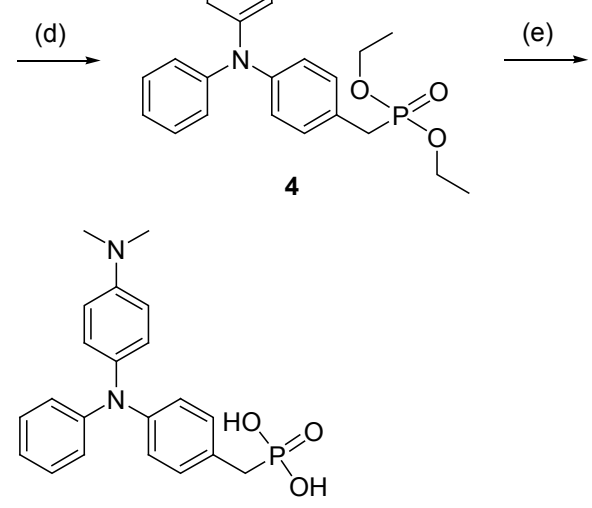

5

图 1 化合物(4-((4-(二甲基氨基)苯基)(苯基)氨基)芐基)膦酸的合成 Figure 1 Synthesis of (4-((4-(dimethylamino)phenyl)(phenyl)amino)benzyl)phosphonic acid

(a) toluene, $\quad \mathrm{Pd}_{2}(\mathrm{dba})_{3} / \mathrm{P}(t-\mathrm{Bu})_{3}, \quad \mathrm{NaOBu}-t, \quad \mathrm{Ar}, 90{ }^{\circ} \mathrm{C}$; (b) toluene, $\mathrm{Pd}_{2}(\mathrm{dba})_{3} / \mathrm{P}(t-\mathrm{Bu})_{3}, \mathrm{Cs}_{2} \mathrm{CO}_{3}, 4$-bromobenzaldehyde, $\mathrm{Ar}, 90{ }^{\circ} \mathrm{C}$; (c) $\mathrm{CHCl}_{3}$ : $\mathrm{CH}_{3} \mathrm{CH}_{2} \mathrm{OH}=3: 1(\mathrm{~V}: V), \mathrm{NaBH}_{4}$; (d) $\mathrm{P}(\mathrm{OEt})_{3} / \mathrm{I}_{2} ;$ (e) $12 \mathrm{~mol} \cdot \mathrm{L}^{-1} \mathrm{HCl}$, reflux

$\left.\mathrm{MHz}, \mathrm{CDCl}_{3}\right) \delta: 7.53(\mathrm{~s}, 2 \mathrm{H}), 7.37 \sim 7.35(\mathrm{~m}, 2 \mathrm{H}), 7.19 \sim$ $7.17(\mathrm{~m}, 1 \mathrm{H}), 7.03 \sim 7.01(\mathrm{~m}, 2 \mathrm{H}), 6.83 \sim 6.68(\mathrm{~m}, 2 \mathrm{H})$, $3.00(\mathrm{~s}, 6 \mathrm{H}), 1.73(\mathrm{bs}, 1 \mathrm{H})$.

\section{1 .2 化合物 2 的合成}

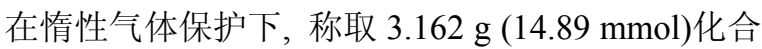
物 1、4 g (21.62 mmol)对溴苯甲醛、 $0.18 \mathrm{~g}(0.20 \mathrm{mmol})$ 三(二亚苠基丙酥)二钯、 $0.0795 \mathrm{~g}(0.40 \mathrm{mmol})$ 三叔丁基 磷、 $4 \mathrm{~g}(20 \mathrm{mmol})$ 碳酸铯加入甲苯中. $90{ }^{\circ} \mathrm{C}$ 下反应 $24 \mathrm{~h}$. 减压除去甲苯, 用氯仿溶解. 过滤之后, 用饱和食盐水 洗涤 3 次. 有机层干燥后过滤, 旋干, 通过柱层析得到 绿色晶体(2.364 g, 50\%). ${ }^{1} \mathrm{H}$ NMR (400 $\left.\mathrm{MHz}, \mathrm{CDCl}_{3}\right) \delta$ : 9.75 (s, 1H), 7.62 (d, $J=8.9 \mathrm{~Hz}, 2 \mathrm{H}), 7.28$ (dd, $J=15.7$, $8.3 \mathrm{~Hz}, 2 \mathrm{H}), 7.18(\mathrm{dd}, J=8.6,1.2 \mathrm{~Hz}, 2 \mathrm{H}), 7.13 \sim 7.05(\mathrm{~m}$, $3 \mathrm{H}), 6.93(\mathrm{~d}, J=8.8 \mathrm{~Hz}, 2 \mathrm{H}), 6.70(\mathrm{~d}, J=9.0 \mathrm{~Hz}, 2 \mathrm{H}), 2.96$ (s, 6H).
2.1 .3 化合物 3 的合成
$0{ }^{\circ} \mathrm{C}$ 下称取 $1.2109 \mathrm{~g}(3.83 \mathrm{mmol})$ 化合物 $2,0.3018 \mathrm{~g}$ 
(7.94 mmol)硼氢化钠溶解在氯仿与乙醇的混合溶剂(体 积比 $3: 1$ )中. $30 \mathrm{~min}$ 后, 转移到室温下反应 $4 \mathrm{~h}$. 向体系 中加入稀盐酸至 $\mathrm{pH}$ 为中性. 用氯仿萃取后, 用饱和食 盐水洗涤 3 次. 有机层干燥后过滤, 旋干得到纯净黄色 固体(1.0967 g, 90\%). ${ }^{1} \mathrm{H}$ NMR (400 MHz, $\left.\mathrm{CDCl}_{3}\right) \delta: 7.11$ $(\mathrm{dd}, J=16.4,9.5 \mathrm{~Hz}, 4 \mathrm{H}), 7.02 \sim 6.88(\mathrm{~m}, 6 \mathrm{H}), 6.82(\mathrm{t}, J=$ $7.3 \mathrm{~Hz}, 1 \mathrm{H}), 6.60(\mathrm{dd}, J=8.7,1.5 \mathrm{~Hz}, 2 \mathrm{H}), 4.48$ (s, 2H), 2.84 (s, 6H), 1.79 (br.s, 1H).

\section{1 .4 化合物 4 的合成}

称取 $1.0967 \mathrm{~g}$ (3.36 mmol)化合物 3 溶解于 $4.9 \mathrm{~mL}$ 亚磷酸三乙酯中, 加入 $0.4267 \mathrm{~g}(3.36 \mathrm{mmol})$ 碘. 搅拌 30 $\mathrm{min}$ 后, 将反应体系转移到室温, 反应 $12 \mathrm{~h}$. 柱层分析得 到产物 4 (0.6041 g, 40\%). ${ }^{1} \mathrm{H} \mathrm{NMR}\left(400 \mathrm{MHz}, \mathrm{CDCl}_{3}\right) \delta$ : $7.22 \sim 6.82(\mathrm{~m}, 11 \mathrm{H}), 6.68(\mathrm{~d}, J=9.0 \mathrm{~Hz}, 2 \mathrm{H}), 4.07 \sim 3.99$ $(\mathrm{m}, 4 \mathrm{H}), 2.92(\mathrm{dd}, J=9.9,3.1 \mathrm{~Hz}, 8 \mathrm{H}), 1.25(\mathrm{t}, J=7.0 \mathrm{~Hz}$, $6 \mathrm{H})$.

\subsection{5 目标产物 5 的合成}

称取 $0.6041 \mathrm{~g}(1.38 \mathrm{mmol})$ 化合物 4 溶解在适量盐酸 中, 回流摚拌 $3 \mathrm{~h}$. 减压除去溶剂, 然后用有机溶剂溶 解. 有机层干燥后过滤, 旋干得到黄色固体(0.3634 g, 69\%). ${ }^{1} \mathrm{H}$ NMR (400 MHz, $\mathrm{CDCl}_{3}$ ) $\delta: 7.62 \sim 7.64$ (m, 2H), $7.32 \sim 7.30(\mathrm{~m}, 2 \mathrm{H}), 7.25 \sim 7.29(\mathrm{~m}, 1 \mathrm{H}), 7.19(\mathrm{~d}, J=12.0$ $\mathrm{Hz}, 2 \mathrm{H}), 7.05 \sim 7.08(\mathrm{~m}, 2 \mathrm{H}), 6.71(\mathrm{~d}, J=8.0 \mathrm{~Hz}, 2 \mathrm{H})$, $6.93(\mathrm{~d}, J=8.0 \mathrm{~Hz}, 2 \mathrm{H}), 4.05$ (s, 2H), 2.96 (s, 6H), 2.50 (s, 2H). HRMS $(\mathrm{FAB}+) \mathrm{M}+\mathrm{Na}$ calcd for $\mathrm{C}_{21} \mathrm{H}_{23} \mathrm{~N}_{2} \mathrm{O}_{3} \mathrm{P}$ : 404.1; found: 404.6. FTIR (ATR) v: 3265 (w), 2961 (m), 2918 (m), 2849 (m), 1592 (m), 1505 (s), 1260 (s), 1164 (m), 1096 (m), 1019 (s), 963 (m), 800 (s), 750 (s), 696 (m) $\mathrm{cm}^{-1}$.

\section{2 化合物的光谱电化学性质}

为了探究化合物的电致变色性能, 我们以 $\mathrm{Ph}_{2} \mathrm{ABPA}-\mathrm{Me}_{2}$ 为电致变色材料制备了工作电极. 驱动工 作电极变色所采用的电极体系为三电极体系, 即参比电 极 (银丝), 对电极 (䈃丝) 和工作电极 (吸附有 $\mathrm{Ph}_{2} \mathrm{ABPA}-\mathrm{Me}_{2}$ 的 $\mathrm{TiO}_{2} / \mathrm{FTO}$ 电极). 将工作电极置于吸收 池中, 以 $0.1 \mathrm{~mol} \cdot \mathrm{L}^{-1} \mathrm{LiClO}_{4}$ 的碳酸丙烯酯溶液为电解 液测得的透射光谱如图 2 所示 $\left(\mathrm{TiO}_{2} / \mathrm{FTO}\right.$ 玻璃和电解液 的透射光谱都已扣除). 当施加 $-1.5 \mathrm{~V}$ 的电压时, 该薄 膜由着色态变为透明态, 对应的响应时间为 $2.5 \mathrm{~s}$; 当施 加 $+2.5 \mathrm{~V}$ 的电压时, 薄膜由透明态转为着色态, 对应 的响应时间为 $10 \mathrm{~s}$. 图中红色虚线是薄膜处于透明态时 的透过率, 此时薄膜的颜色为浅黄色 (见图 2b), 从曲线 上可以看出在 $550 \mathrm{~nm}$ 以前, 透过率随着波长的增大而 增加, $550 \mathrm{~nm}$ 时透过率达到最大值; 从 $550 \mathrm{~nm}$ 到 800 $\mathrm{nm}$ 透过率基本保持在 $80 \%$ 左右. 黑色实线是薄膜处于 着色态时的透过率, 此时薄膜的颜色为墨绿色 (见图 2a), 从曲线上可以看出, 薄膜在着色状态下的透过率在 500 $\mathrm{nm}$ 以前随着波长的增大而增加, 从 $500 \mathrm{~nm}$ 到 $630 \mathrm{~nm}$
附近随着波长的增大而减少，随后又经历增加过程一直 到波长 $800 \mathrm{~nm}$ 处. 在这里, 透过率对比度被定义为:

$$
\Delta T=T^{\mathrm{t}}(\lambda)-T^{\mathrm{c}}(\lambda)
$$

$T^{\mathrm{t}}(\lambda)$ 和 $T^{\mathrm{c}}(\lambda)$ 分别是指在固定波长处透明态和着色态的 透过率 ${ }^{[11]}$. 在完全褪色和着色状态下, 该薄膜的透过率 对比度在 $626 \mathrm{~nm}$ 处达到最大, 为 $64.2 \%$.

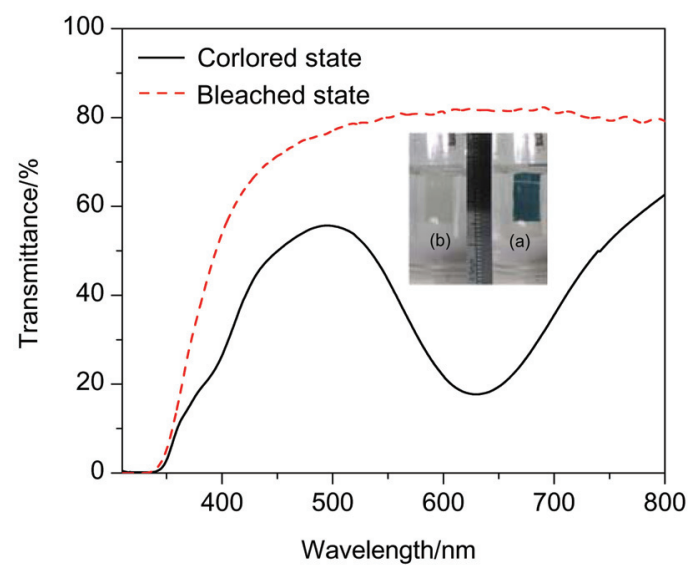

图 $2 \mathrm{Ph}_{2} \mathrm{ABPA}-\mathrm{Me}_{2}$ 薄膜在两种状态下的透射光谱. 图中照片为工作 电极在不同电压下的颜色状态: (a) $+2.5 \mathrm{~V}$ 下为墨绿色; (b) $-1.5 \mathrm{~V}$ 下为浅黄色

Figure 2 Transmittance spectra of $\mathrm{Ph}_{2} \mathrm{ABPA}-\mathrm{Me}_{2}$ film in the visible region. Pictures of the working electrode at different coloration states: (a) dark green at $+2.5 \mathrm{~V}$; (b) light yellow at $-1.5 \mathrm{~V}$

\section{3 自供能电致变色器件的光伏性质}

作为自供能电致变色材料, 除了具有电致变色性能 外, 光伏性能也不可或缺. 为了探究 $\mathrm{Ph}_{2} \mathrm{ABPA}-\mathrm{Me}_{2}$ 化合 物的光伏性能, 我们将其作为染料制作成器件, 其结构 如图 3 所示. 该器件的面积为 $0.36 \mathrm{~cm}^{2}$. 在 $\mathrm{AM} 1.5 \mathrm{G}$ 一 个标准太阳光照射下, 我们得到了器件的光伏效率曲线 如图 4 所示. 由图 4 可知, 该器件的短路电流为 1.32 $\mathrm{mA} / \mathrm{cm}^{2}$, 开路电压为 $0.44 \mathrm{~V}$, 填充因子为 0.54 , 计算得 到光电转化效率为 $0.32 \%$. 该器件的填充因子较低, 说 明器件的电阻较大 ${ }^{[12]}$. 因此, 优化器件结构, 降低器件 电阻以提高光电转化效率成为我们接下来的主要工作.

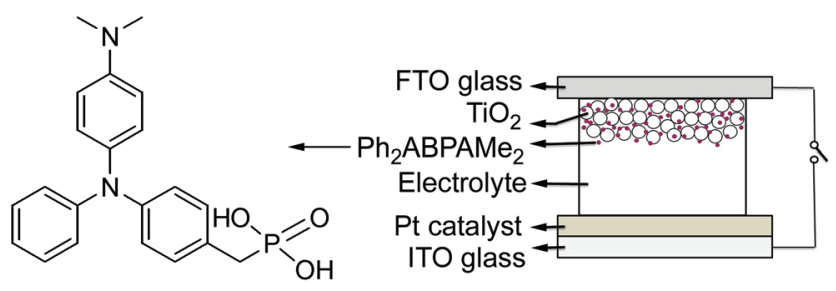

图 3 自供能电致变色器件结构图

Figure 3 Schematic of self-powered electrochromic device

\section{3 结论}

本文设计合成的新型三苯胺衍生物 $\mathrm{Ph}_{2} \mathrm{ABPA}-\mathrm{Me}_{2}$ 具有双重性能, 即光电转换和电致变色性能. 在 $626 \mathrm{~nm}$ 处, 不同状态下 $\mathrm{Ph}_{2} \mathrm{ABPA}-\mathrm{Me}_{2}$ 薄膜的透过率对比度最 


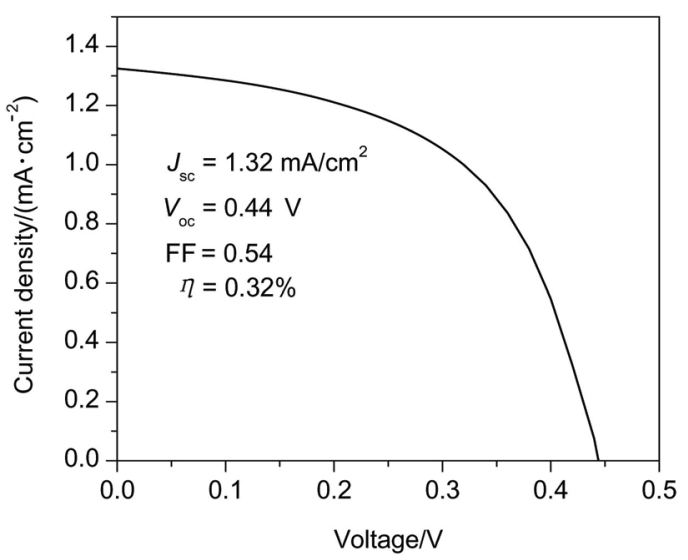

图 4 自供能电致变色器件在 $\mathrm{AM} 1.5 \mathrm{G}$ 一个标准太阳光照射下的光电 流-电压曲线

Figure 4 Photocurrent-voltage $(I \sim V)$ curve of self-powered electrochromic device under 1 sun AM1.5G illumination

大可以达到 $64.2 \%$; 该化合物同时具有光电转换性能, 但是光电转换效率比较低, 因此优化器件结构以提高光 电转换效率是我们下一步的工作. 可以预测如果基于该 材料的自供能电致变色器件可以实现自供能, 不仅可以 节约能源, 优化环境, 其应用前景也将十分可观, 比如 应用于建筑、汽车、显示器等领域.

\section{4 实验部分}

\section{1 试剂材料}

苯胺, 碳酸铯, 无水硫酸钠, 盐酸购自国药; 三叔 丁基膦 $\left(1.0 \mathrm{~mol} \cdot \mathrm{L}^{-1}\right.$ 甲苯溶液), 亚磷酸三乙酯购自 Aladdin (上海); 4-溴- $N, N$-二甲基苯胺, 对溴苯甲醛购自 Sigma-Aldrich (上海); Pt-催化剂 T/SP, 电解液 Iodolyte AN-50, Ti-Nanoxide HT 购自 Solaronix (Aubonne VD, Switzerland); 碳酸丙烯酯(PC), 无水高氯酸锂 $(99 \%)$, 叔 丁醇钠, 三(二亚苄基丙酮)二钯购自 Alfa Aesar (Ward Hill, MA, USA). 四氢呋喃、PC 和甲苯在使用之前需进 行除水操作.

\section{2 电极的制备及自供能电致变色器件的组装}

\subsection{1 对电极的制备}

将 Pt 催化剂凝胶涂抹到透明导电基底 ITO (氧化铟 锡)导电面, 在 $260{ }^{\circ} \mathrm{C}$ 下加热 $10 \mathrm{~min}$ 后取出, 冷却至室 温, 得到对电极.

\subsection{2 工作电极的制备}

采用刮刀法在透明导电基底 $\mathrm{FTO}$ (氟掺杂氧化锡)导 电面涂覆二氧化钛薄膜, 在 $450{ }^{\circ} \mathrm{C}$ 下加热 $30 \mathrm{~min}$ 后, 置 于 $\mathrm{Ph}_{2} \mathrm{ABPA}-\mathrm{Me}_{2}$ 的氯仿溶液中吸附 $24 \mathrm{~h}$, 得到工作电 极.

\subsection{3 自供能电致变色器件的组装}

将工作电极、电解液 Iodolyte AN-50 和对电极进行 组装, 得到自供能电致变色器件. 具体如下: 先将双组 分胶粘在工作电极薄膜边缘，并留下一个注射口; 将对 电极盖在工作电极上，使对电极的 Pt 膜与工作电极的 纳米二氧化钛膜薄膜相对, 使用夹具夹紧两片电极. 待 双组分胶凝固后, 取下夹具, 利用 $1 \mathrm{~mL}$ 注射器将电解 液通过事先留下的注射口注入两片电极之间, 注满后再 用双组分胶将注射口封住. 组装完毕后得到自供能电致 变色器件.

\section{3 仪器设备}

$\mathrm{Ph}_{2} \mathrm{ABPA}-\mathrm{Me}_{2}$ 的光谱电化学性质是用紫外可见分 光光度计 (V-670; JASCO, Tokyo, Japan)结合电化学工作 站(CHI 660D, 上海)测得的; 自供能电致变色器件的光 伏性质是用太阳能模拟器(OTENTO-SUN II; Bunkoukeiki Co., Japan)测得的.

\section{References}

[1] Bechinger, C.; Ferrer, S.; Zaban, A.; Sprague, J.; Gregg, B. A. Nature 1996, 383, 608 .

[2] Yang, S. W.; Zheng, J. M.; Li, M.; Xu, C. Y. Sol. Energy Mater. Sol. Cells 2012, 97, 186.

[3] Yuan, J. G.; Zhang, Y. Z.; Le, J.; Song, L. X.; Hu, X. F. Acta Chim. Sinica 2005, 63, 1884. (袁嘉国, 章俞之, 乐军, 宋力昕, 胡行方, 化学学报, 2005, 63, 1884.)

[4] Yu, M. X.; Chang, L. C.; Lin, C. H.; Duan, J. P.; Wu, F. I.; Chen, I. C.; Cheng, C. H. Adv. Funct. Mater. 2007, 17, 369.

[5] Yue, Y.; Kang, J.; Yu, M. Dyes Pigm. 2009, 83, 72.

[6] Zeng, W. D.; Cao, Y. M.; Bai, Y.; Wang, Y. H.; Shi, Y. S.; Zhang, M.; Wang, F. F.; Pan, C. Y.; Wang, P. Chem. Mater. 2010, 22, 1915.

[7] Lee, D. H.; Lee, M. J.; Song, H. M.; Song, B. J.; Seo, K. D.; Pastore, M.; Anselmi, C.; Fantacci, S.; De Angelis, F.; Nazeeruddin, M. K.; Graetzel, M.; Kim, H. K. Dyes Pigm. 2011, 91, 192.

[8] Hua, J. L.; Li, B.; Meng, F. S.; Ding, F.; Qian, S. X.; Tian, H. Polymer 2004, 45, 7143 .

[9] Li, B.; Li, Q. A.; Liu, B.; Yue, Y. F.; Yu, M. X. Dyes Pigm. 2011, 88, 301.

[10] Yen, H. J.; Lin, H. Y.; Liou, G. S. Chem. Mater. 2011, 23, 1874.

[11] Ma, C.; Taya, M.; Xu, C. Y. Electrochim. Acta 2008, 54, 598.

[12] Thompson, B. C.; Kim, Y. G.; McCarley, T. D.; Reynolds, J. R. J. Am. Chem. Soc. 2006, 128, 12714. 